\title{
Node importance evaluation method in wireless sensor network based on energy field model
}

\author{
Qindong Sun ${ }^{1,2^{*}}$, Yimin Qiao ${ }^{1}$, Jiamin Wang $^{3}$ and Si Shen ${ }^{1}$
}

\begin{abstract}
The stability degree of key nodes is an important indicator of wireless sensor network performance. Appropriate node importance evaluation method is a precondition for the identification of key node and the analysis on network stability. The current methods based on average length and network density are unable to make real-time evaluation on nodes in practical application. Thus, this paper puts forward a node importance evaluation method in wireless sensor network based on energy field model. Based on the data exchange at the network layer in wireless sensor network, this approach analyzes the node properties and correlation among nodes with energy field and graph theory, which shows the influence result of key nodes in wireless sensor network. Therefore, the node influence can be worked out so as to evaluate the importance of node in wireless sensor network. The experimental results show that, compared with the current node importance evaluation methods of wireless sensor network, the approach proposed in this paper not only is able to evaluate the node importance without affecting the operation of network but also shows the dynamic change in node importance.
\end{abstract}

Keywords: Energy field, Wireless sensor network, Importance evaluation of node

\section{Introduction}

With the feature of flexible network settings and convenient network building, the wireless sensor network, in which the sensors communicate with each other wirelessly, is able to perceive, collect, and process the information in the network monitoring area [1]. Wireless sensor network has the capability of being selforganized, lots of single nodes formed up the multi-hop ad hoc network which is used to transmit data by initial communication and negotiation [2]. The wireless sensor network has no preexisting physical infrastructure that restricts the topology [3]. It can be applied in such fields as military, agriculture, meteorology, biology, and inaccessible areas to collect information [4]. With the advent of the Internet of things, wireless sensor network is gradually pervading every aspect of people's life, and it has significant value of scientific research and broad

\footnotetext{
* Correspondence: sqd@xaut.edu.cn

'School of Computer Science and Engineering, Xi'an University of

Technology, Xi'an 710048, China

${ }^{2}$ Shaanxi Key Laboratory of Network Computing and Security, Xi'an University of Technology, Xi'an 710048, China

Full list of author information is available at the end of the article
}

application prospect [5]. With the wide application of wireless sensor network, the demands on the reliability of its communication network are also getting higher and higher. Influenced by factors such as energy, environment, and network structure, some nodes of network may be damaged or may fail, etc., these unexpected situations will impact the performance of the network [6]. The failure of key nodes may even cause the networks paralysis. Therefore, evaluating the importance of each node and finding out the key nodes in network contribute to the maintenance of important nodes and thus improve the survivability of network.

Considerable studies have been carried out on the abovementioned problem. There are many methods to evaluate the mode influence in wireless sensor network. Most of the existing methods to evaluate the node importance are based on such indicators as the average path length, the network density, the network diameter, and the electric quantity of node. These methods are using the node-deletion method by removing the key nodes during evaluation and observing the changes (such as network disconnection) of energy consumption 
in network after the key node is removed or failed. However, these methods are difficult to implement in practical application due to restrictions of actual situation. Besides, some studies evaluate the node importance in wireless sensor network with statistical methods. However, these studies have certain limitations that the data transmission process in network is not considered while evaluating the influence of nodes.

This paper proposes a node importance evaluation approach in wireless sensor network based on energy field model which evaluates the node importance by analyzing the data transmission status among associated nodes at the network layer in the wireless sensor network. Inspired by the field theory in physics, the approach studies the changes of node influence in wireless sensor network from the perspective of energy field. Besides, the network topology may change due to the added nodes for improving detection precision in practical application. That is to say, some nodes with higher importance may become less important after the change. Therefore, this approach gives full consideration to the dynamic change of nodes in importance evaluation. The advantage of the proposed approach is that the node importance is evaluated without affecting the physical structure of the network itself, which can well reflect the change of the node importance in the process of dynamic change and reduces the workload of node importance evaluation process.

The rest of the paper is arranged as follows. Some research methods in relevant fields are introduced and analyzed in Section 2. Elaboration is made on the importance evaluation approach of node in wireless sensor network based on energy field model in Section 3. Simulation experiments are conducted in Section 4 so as to verify the approach proposed in Section 3, and comparative analysis with other two relevant methods is carried out in this section.

\section{Related works}

Researches on complex network theory have provided some new ideas for the study on the node importance evaluation in wireless sensor network. In [7] the authors proposed a key node identification method based on the volume of aggregated nodes. The method of node importance considered the connection characters of the nodes. In this paper, the node importance referred to the weighted sum of the self-importance of the node itself and edges that are connected with the node. In [8], the authors proposed a semi-local centrality measure which evaluated the importance of a node through a susceptible-infected-recovered (SIR) model based on the propagation velocity of a node message and the number of nodes which receive message; this effective ranking method is capable to be applied in large-scale networks.
Considering the deficiency of traditional indicators in key node positioning, in [9], the authors proposed a key node identification method which uses the communication probability of a node and $k$-shell decomposition method to evaluate the importance of a node. By using multiple indicators to evaluate node importance although these methods can obtain more precise result, there are limitations in these methods with regard to characteristics of the wireless sensor network such as limited energy and operating rate. In [10], the authors proposed a method based on finite number of objects and ideal objective and evaluated the importance of each node in network through TOPSIS technique which is utilized to aggregate the multi-attribute to obtain the result. In [11], the authors brought in webpage grade vectors in PageRank algorithm to rank the nodes and thus evaluated the node importance. In [12], the authors proposed an evidence-theory-based measurement which could weigh both the degree and strength of every node in a weighted network. The node influence could be represented by basic probability assignment (BPA), and the measure in this paper is determined by the combination of BPAs. Inspired by the field theory in physics, in [13], the authors proposed a topological sorting algorithm in which each node in the network was seen as a material particle. The potential field around one node itself and the interaction of all nodes forms a topological field. Through the definition and calculation of potential topological weight of each node, more precise ranking results, which could show the importance of each node in network, were obtained. However, these methods can only rank the nodes in network with some specific indicators but fail to consider the life period of node influencing on the entire network. In [14], the authors proposed a key node determining algorithm based on local information and energy factors. The algorithm proposed a model based on the node degree and the local information of neighboring nodes and evaluated the node importance through the surplus energy of node together with the energy consumption change in network after node failure. However, this algorithm only considered the energy of node and data transmission in wireless sensor network, but the relation between nodes in node importance evaluation process is not considered.

The abovementioned methods are of significant reference value for our node importance evaluation method. The previous studies on the node importance evaluation mainly focus on the analysis of one certain factor or multiple factors or are based on the combination of complex network theory, statistics, and other theories. The essential function of nodes in the network is to transmit the data; however, the studies of the data transmission at network layer in wireless sensor network are rarely considered. Therefore, the determining results are 
unable to reflect the dynamic change process in wireless sensor network fully and faithfully and thus desired effects cannot be achieved in later network maintenance.

\section{Algorithm analysis}

In physics, many physical phenomena are related to the field model which describes the interaction relationship between different objects. The interaction between two different entities is manifested by the operation of the energy. The physical properties of field can be used to depict the physical phenomenon in field space. The relationships between different variables reveal the ways of energy conversion in the field [15]. Therefore, the features of the field can be used to solve similar practical problems.

In the preliminary work, we put forward an influence calculation model of users in online social network based on field theory by using the data of Sina microblog. A comparative analysis was carried out on the influence calculation model through experiments. The experimental results showed that this model had a better performance in user classification [16]. This paper proposes a node importance evaluation method in wireless sensor network based on the energy field model, in which the influence transfer refers to the data transmission between different nodes and the transmission process refers to the process of influence transferring along the network topology. The process is regarded as the accumulation of node influence. The relationship between nodes and the node influence is analyzed with the characteristics of nodes in the process of data transmission. The node influence in network generates or attenuates when the data transmit among nodes. In other words, when a node sends data, a certain part of energy of the node is consumed. Finally, the importance of the node is evaluated by the influence of the nodes which generated in the process of data transmission in wireless sensor network.
All the nodes in the target network which are set in indirect structure have the same functional characteristics. When $N_{i}$ sends a data message, its related $N_{j}$ will forward the message. If $N_{j}$ is not the destination node of the message, $N_{j}$ will forward the message. It means that $N_{i}$ has exerted an influence on $N_{j}$ during data transmission. In the forwarding process, the influence of the two nodes $N_{i}$ and $N_{j}$ will increase. Therefore, the influence of $N_{i}$ on $N_{j}$ is larger than that of $N_{j}$ on $N_{i}$. $N_{j}$ receives message from node $N_{i}$, which shows the influence of node $N_{i}$ indirectly. In other words, $N_{j}$ will get influence from node $N_{i}$ while forwarding the message received from $N_{i}$. Meanwhile, $N_{j}$ will feed back some influence to node $N_{i}$. When the message forwards through nodes one after another, the influence will transfer and generate feedback constantly between relevant nodes, as shown in Fig. 1.

In wireless senor network, the operation of the energy should be obtained when data is transferred between different nodes. Then, the influence of the corresponding nodes is calculated by calculating the parameters in the data transmission process in the network layer. For any two nodes, $N_{i}$ sends the message and $N_{j}$ receives the message. The energy consumed for transmitting $k$ bit data is expressed through the universal wireless communication energy consumption model as follows [17]:

$$
E_{\text {send }}(k, d) E_{\text {elec }} k+\varepsilon_{\text {amp }} k d_{\mathrm{ij}}^{2}
$$

The energy consumed for receiving $k$ bit data is:

$$
E_{\text {receive }}(k)=E_{\text {elec }} k
$$

where $E_{\text {elec }}$ is the transmission coefficient of radio frequency, $\varepsilon_{\mathrm{amp}}$ is the amplification coefficient of sending device, $d_{\mathrm{ij}}$ is the data transmission radius of the node. The congestion of node can be written as follows:

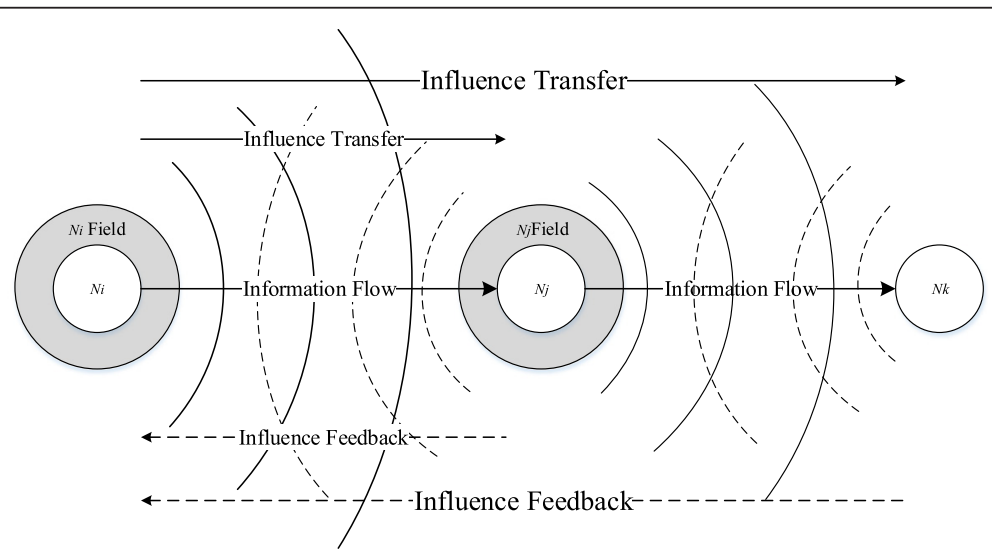

Fig. 1 The schematic diagram of influence transfer 


$$
J=\frac{C_{\text {dsend }}}{C_{\text {receive }}+C_{\text {send }}}
$$

where $C_{\mathrm{dsend}}$ is the number of unsent messages of the node, $C_{\text {receive }}$ is the number of received messages, and $C_{\text {send }}$ is the number of sent messages.

The distance between $N_{i}$ and $N_{j}$ is 1 ; thus, the influence of $N_{i}$ can be expressed as $I_{r=1}$. When the distance between $N_{i}$ and $N_{j}$ is larger than 1 , the influence of $N_{i}$ is expressed as $I_{r>1}$. The influence in the expressions generates in one data acquisition period. The influence generated during message transmission is:

$$
\left\{\begin{array}{l}
I_{r=1}=f_{1}\left(N_{i}, C_{r}\right) \\
I_{r>1}=f_{2}\left(I\left(N_{f a}\right), r\right)
\end{array}\right.
$$

where the value of $I_{r=1}$ is related to the node properties and the number of messages $C_{r}$ that $N_{j}$ forward from $N_{i}$. The value of $I_{r>1}$ depends on the distance $r$ between $N_{j}$ and $N_{i}$ as well as the influence of father $N_{f a}$ (the node near sink is father node).

When $r=1$, the formula of influence derived from field theory is:

$$
I_{r=1}=G \frac{C_{r}}{C_{p}}\left(E_{\text {send }}+E_{\text {receive }}\right)
$$

where $C_{r}$ is the number of messages $N_{j}$ forward from $N_{i}$ in one data reception period and $C_{p}$ is the number of messages received from $N_{j}$ in one data reception period. The ratio of $C_{r}$ to $C_{p}$ refers to the correlation coefficient of $N_{j}$ to $N_{i}$. G refers to the density of nodes around $N_{i}$ in one data reception period. $E_{\text {send }}$ refers to the energy of $N_{i}$ consumed for sending $k$ bit data. $E_{\text {receive }}$ refers to the energy of $N_{j}$ consumed for receiving $k$ bit data. $G$ can be expressed as follows:

$$
G=\frac{K_{i}}{K_{\max }}
$$

where $K_{i}$ refers to the actual sides connected with $N_{i}$ and $K_{\max }$ refers to the possible maximum sides connected with $N_{i} . k_{i}$ is the degree of $N_{i}$. Then, $K_{\max }$ can be expressed as:

$$
K_{\max }=\frac{k_{i}\left(k_{i}-1\right)}{2}
$$

When $r>1$ :

$$
I_{r>1}=I\left(N_{f a}\right) P\left(J_{f a}, r\right)
$$

where $I\left(N_{f a}\right)$ is the influence of the father nodes and $P$ is the forward probability. The larger the surplus energy, the higher the probability of becoming a forwarding node. $J_{f a}$ is the congestion rate of the father node. Considering the message may impair in forwarding, the forward probability is:

$$
P=\left(P_{E} J_{f a}\right)^{r}
$$

where $P_{E}$ refers to the surplus energy ratio of the node:

$$
r=\left|e^{\mu d_{i j}}\right|
$$

where $\mu$ is the weighted value of the range attenuation. The influence of $N i$ is:

$$
I=\sum_{n} I_{r=1}+\sum_{m} I_{r>1}
$$

where $n$ refers to the number of nodes which are away from node $N_{i}$ with the distance $r$ equaling 1 and $m$ refers to the number of nodes causing the influence with the distance $r$ greater than 1 . The influence of node $N_{i}$ could be denoted by the influence of these nodes in wireless sensor network, as shown in Fig. 2.

There are four steps to evaluate node importance based on energy field model:

(1) Calculate the energy consumption in the process of data transmission in the network

(2) Choose the parameters which are related to the node in the process of data transmission

(3) Under the given experimental environment conditions, calculate the $I_{r=1}$ and $I_{r>1}$

(4) Calculate the influence of all nodes in the whole network by expression (11) and find out the influential nodes according to the results

\section{Experimental results analysis}

\subsection{The evaluation results of important nodes}

In order to verify the node importance evaluation approach, we have carried out some simulation experiments in this section. The parameters in modeling are set as follows: $E_{\text {elec }}=50(\mathrm{~nJ} / \mathrm{bit}) ; \varepsilon_{\mathrm{amp}}=100\left(\mathrm{pJ} / \mathrm{bit} / \mathrm{m}^{2}\right)$; the maximum transmission radius of wireless node is 200; the nodes are evenly distributed in a $100 \times 100 \mathrm{~m}^{2}$. For the convenience of calculation, 100 nodes which are not on the boundary of the square are selected. The data quantity sent by node each time and the times of data sending and receiving are random. The influence of nodes which are caused by the distance $r=1$ from node $N_{i}$ is calculated through expression (5). The normalized results are shown in Fig. 3. In Fig. 3, it can be seen that the influence distribution of node is relatively average and the influence values is mainly distributed between 0 and 0.5 . The influence of nodes which are caused by the distance $r$ greater than 1 is calculated through expression (8). The normalized results are shown in Fig. 4. Compared with the result in Fig. 3, the influence values in Fig. 4 are smaller and mainly distributed between 0 and 0.3 It should be pointed out that the iteration of influence of father nodes and the correlation between the 


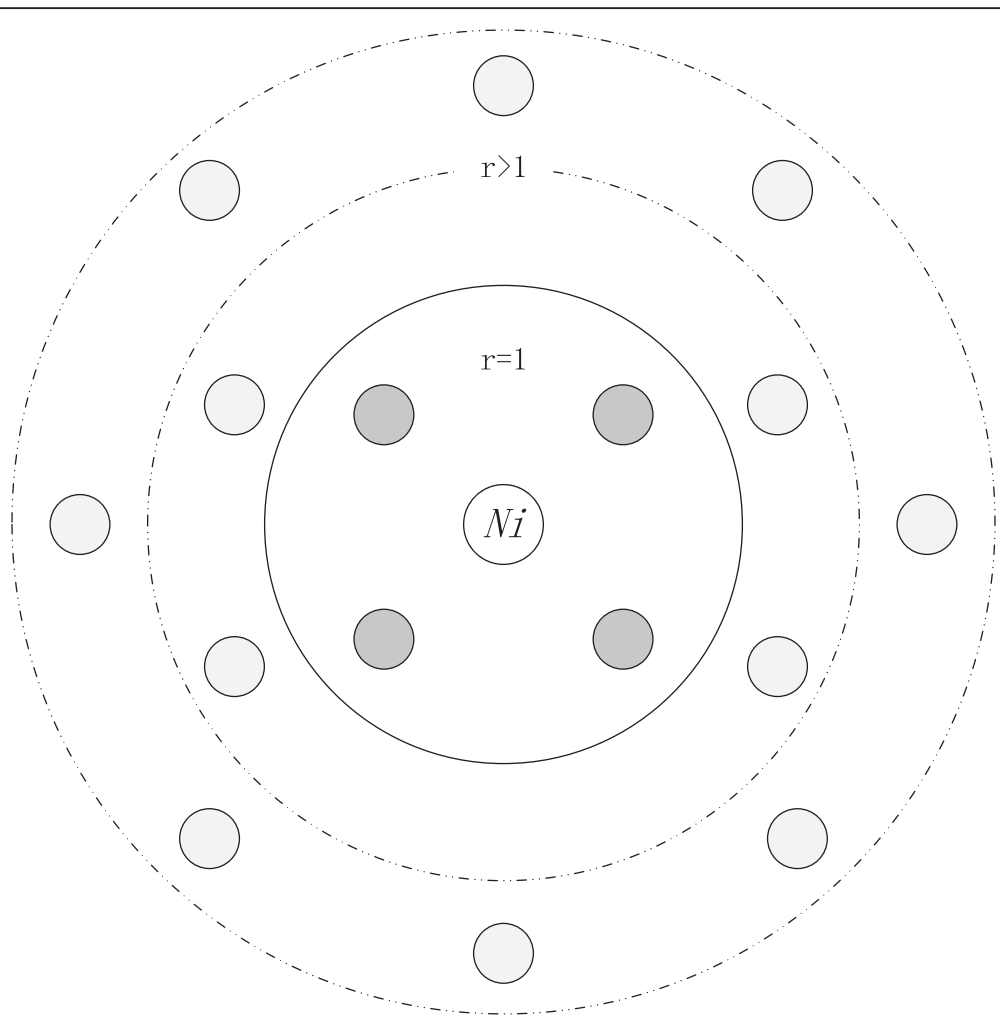

Fig. 2 The schematic diagram of node influence calculation

forwarding probability and distance in data transmission should be considered. It can be known from the two figures that the distribution of node influence is uneven, and the nodes with bigger value are more likely having higher influence.

After working out the influence of nodes related to $N_{i}$, the influence of $N_{i}$ in the entire network can be obtained through expression (11). Considering the iteration in calculation, the initial influence of $N_{\mathrm{i}}$ and that of nodes in distance 1 with $N_{\mathrm{i}}$ should be excluded. The influence distribution of nodes in the entire network can be obtained after calculating the influence of nodes one after another. The normalized results are shown in Fig. 5.

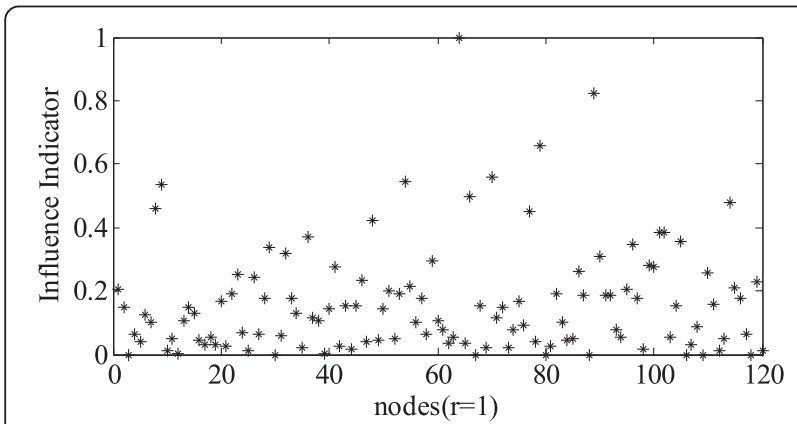

Fig. 3 The influence distribution of nodes with the distance $r=1$ with $N_{i}$ in one data reception period
The analysis results of the selected 100 nodes are shown in Fig. 6, where the cross refers to the number of messages passed $N_{j}$ in one data reception period, i.e., $C_{p}$, and the triangle refers to the number of messages $N_{j}$ forward from $N_{i}$, i.e., $C_{r}$. It can be known from Fig. 5 that the distribution of most nodes is similar to those of $C_{p}$ and $C_{r}$ except several nodes. That is, because the deployment of nodes is different in the actual network, the network is a self-organizing network and the attributes factors of the node (such as spatial location, signal interference, the remaining energy) and the nodes with strong load capacity (such as the node communication efficiency is high, the signal interference is weak, more

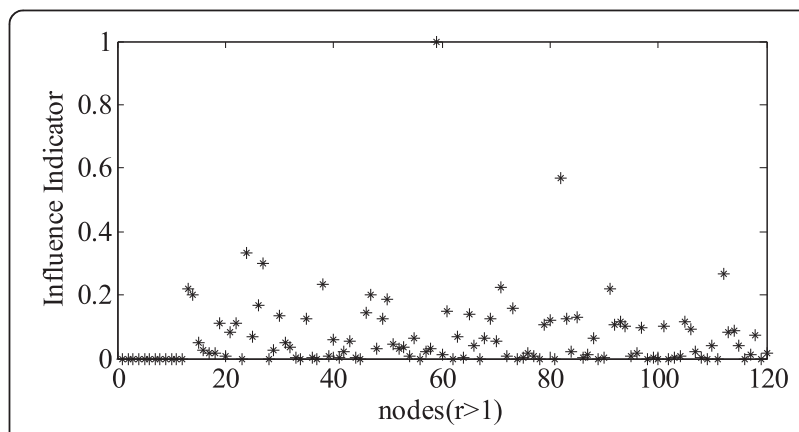

Fig. 4 The influence distribution of nodes with the distance $r>1$ with $N_{i}$ in one data reception period 


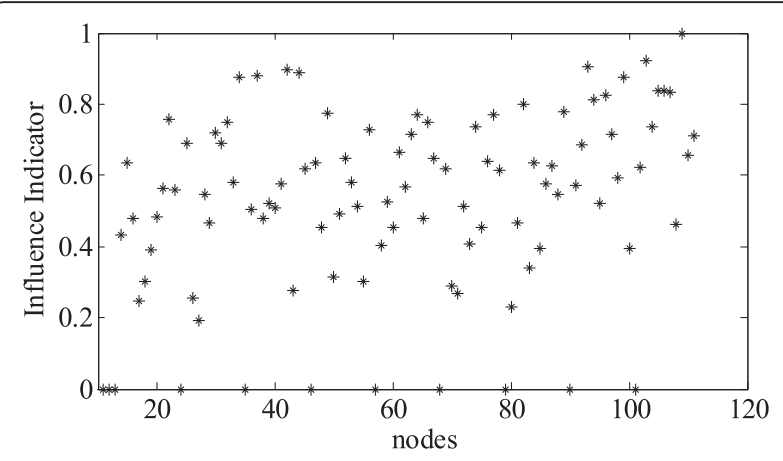

Fig. 5 The influence distribution of node

remaining energy) will assume more data flow to ensure the data transmission while other nodes is able to play the role of the nodes mentioned above at any time. These nodes only serve as data channel and has strong substitutability in the network but contribute little. Therefore, the nodes with large carrying capacity may be small in influence. Through the above experimental analysis, the nodes in the network can be divided into three categories and make different maintenance strategies for different type of the nodes. The first is the nodes with larger influence should be focus on the protection, the second is the nodes with large carrying capacity but small in influence need to carry out routine maintenance, and the third is the nodes with little influence and carrying capacity should have planned sleep and be activated when needed.

\subsection{Efficiency analysis of algorithm}

We have carried out comparative analysis with some existing node influence evaluation methods such as cluster contraction [18], energy aware [19]. The node importance evaluation method based on the cluster contraction principle is using certain rules to find the cluster structure in the network. The nodes can be divided into two categories, one is the organ nodes, and the other is the joint nodes that does not belong to any cluster. The joint nodes play the role of connecting organ

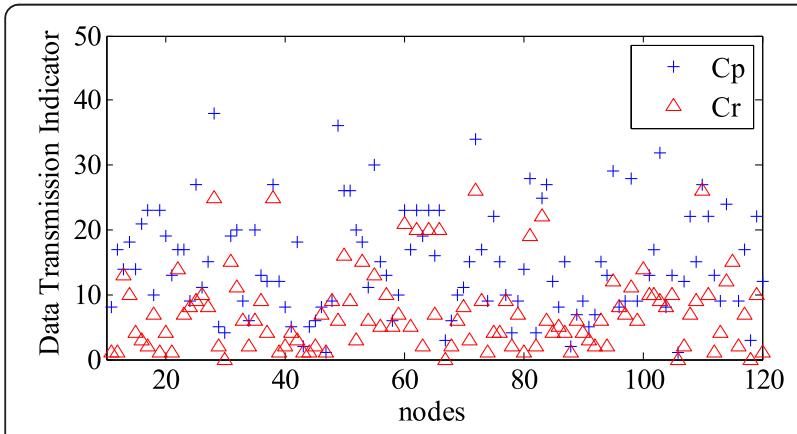

Fig. 6 The data transmission distribution of node nodes. It is possible to cause the network paralysis when a joint node failure. The organ nodes distribution density is bigger, and there will have redundant nodes to replace it when an organ node failed and has little damage to the network. The backbone network will be obtained by shrinking organ nodes and then evaluating the importance of the joint node in the network. The node importance evaluation method based on energy aware is the study of using the residual energy information as the practical basis of algorithm design. This method combines the node degree with the energy consumption and other related factors in the network and evaluates the node importance by calculating the residual energy of nods and the total energy consumption after corresponding node fails in the network. It can be known from the experimental results that the influence obtained through the node importance evaluation model based on energy field model is higher than that obtained through the other two models, as shown in Fig. 6.

Some simulation experiments are conducted for the node influence obtained through the three methods. The influence in several data reception periods calculated through the method based on energy field model shows more dramatic change than that calculated through the methods based on cluster contraction and energy aware, as shown in Fig. 7. That is because in the current simulation experiments, the data quantity sent and received by nodes is usually fixed. Thus, it is unable to show the influence of sensor errors, sensor noise, and error of transmission, leading to the differences in the data transmitted by nodes in the same sensor area. Considering the change in network topology caused by the variation of node amount, our method is able to show the dynamic changing process of the node influence in network as well as analyze the variation trend of node importance through the change detected in data transmission. Hence, the maintenance strategy of the nodes should be changed when the influence of nodes changed, this dynamic maintenance process can greatly enhance the monitoring time of the whole network., as shown in Fig. 8.

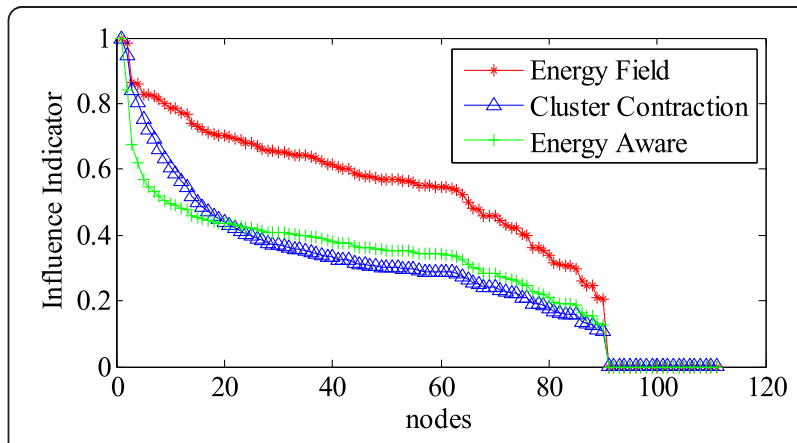

Fig. 7 The comparison among the results from the node influence evaluation methods based on energy field model, cluster contraction, and energy aware 

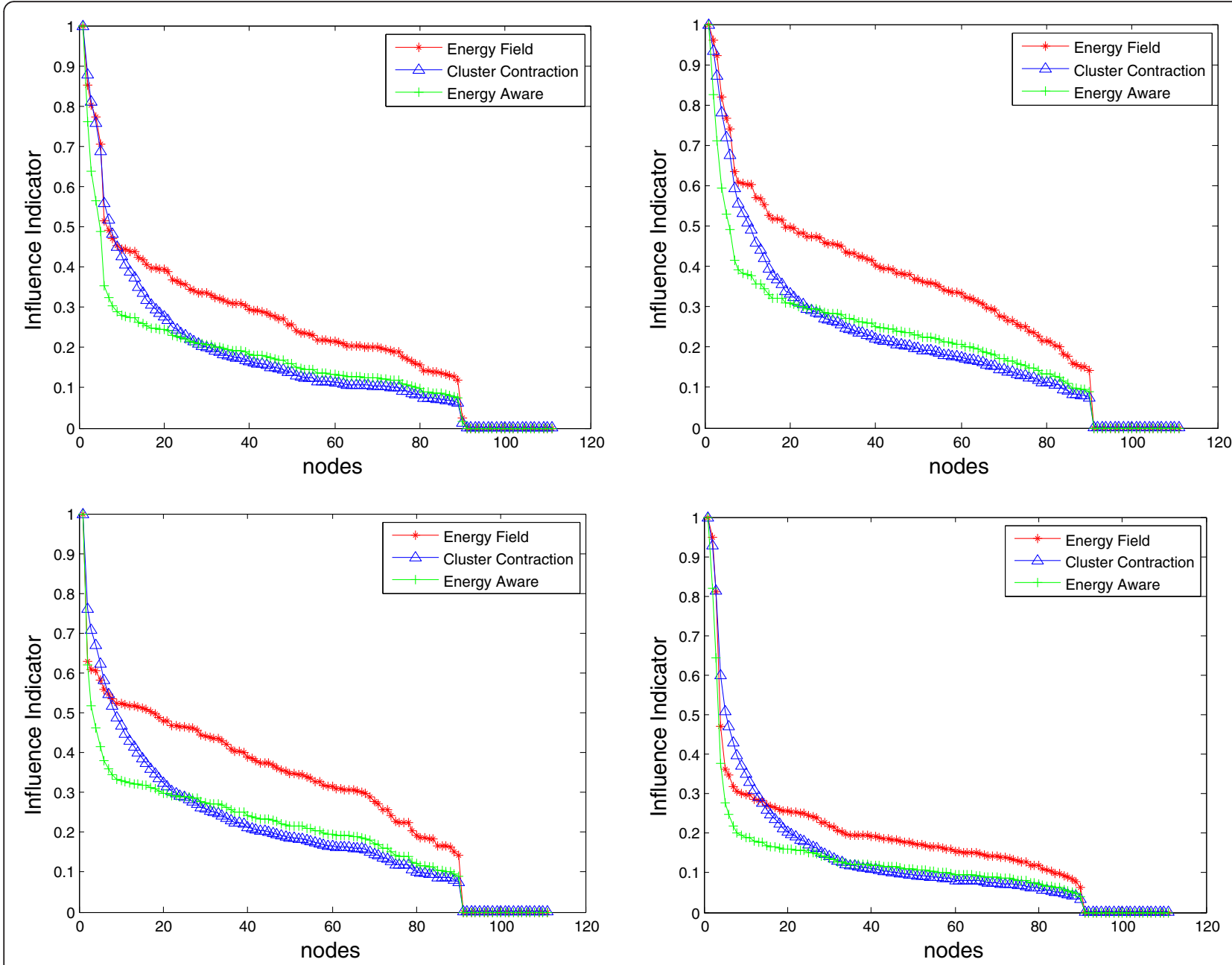

Fig. 8 Comparison of several simulation experimental results

\section{Discussion}

The method proposed in this paper is based on the energy field model which concerns node characteristics to evaluate node importance as well as the data transmission procession between different nodes. Different from other methods, our model can express the relationship between the node influence and data transmission and depict the dynamic change of node importance by our model in wireless sensor network. Hence, in solving some relevant problems, our model is advantageous. Besides, the model proposed in this paper is flexible, which can be adjusted according to different problems in practical situation. Aiming at some research points in wireless sensor network, our method has advantage in solving the following problems.

\subsection{Energy management}

At present, the energy management in wireless sensor network is mainly divided into three kinds of methods, which are the power-aware routing protocols, the topology protocols, and the sleeping management. The model based on power-aware routing protocols is used to uniform the energy in whole network and effectively prolong the survival time of key nodes, but it cannot fully depict the details of data transmission process between nodes. The model based on topology protocols is used to reduce the transmission energy consumption of nodes by controlling the communication links between nodes and transmitting rage of nodes in the network in order to prolong the life cycle of the whole network and improve its network efficiency, but it lacks consideration of the role of important nodes and corresponding energy consumption in the network. The model based on sleeping management to prolong the network lifetime by keeping the redundant nodes to sleep but may cause data loss and less accuracy. Based on this model, the dynamic node importance evaluation can provide help for energy management, which makes it more accurate and more suitable for practical applications. 


\subsection{Network survivability}

According to the characters of the network, network survivability is generally use as survivability measurement as the basis and establishes the survivability analysis model based on the probability theory and graph theory, etc. The node importance evaluation as an important aspect in network survivability has many indicators such as degree, betweenness, compactness, feature vector. The degree is simple, intuitive, and easy to calculate but only reflects the local characteristics. The betweenness evaluates the node importance from the point of view of flow, which can reflect the dynamic characteristics of the network, but is not suitable for practical applications because of its high computational complexity. The compactness reflects the degree of centrality of nodes based on the topology of network which has certain limitations on some kinds of network. The feature vector is simplistic of the actual situation by taking the importance of neighbor nodes into account. Based on the energy field model, the relationship between different nodes is well considered in this method which is useful for some other methods and is more applicable to actual situation.

\section{Conclusions}

In this paper, we analyzed the node interactions based on the influence transfer effect in wireless sensor networks and found that traditional models have limitations in describing a dynamic process in wireless sensor networks. First, these methods do not account for data transmission mechanisms and transmission probability that can affect the node evaluation effects. Second, the node failures and other factors often cause the change of the network, and these methods cannot adapt to evaluate node influence in a dynamic network environment. Third, these methods have a rough classification of the nodes in wireless sensor network and cannot identify the nodes with large data flow but have limited influence in the network.

Node importance evaluation is a precondition for the identification of key nodes and the analysis on network stability. This paper has proposed a node importance evaluation approach based on energy field model. The idea is that the influence transfers and generates feedback constantly in the process of data transmission which creates a field and interaction with related nodes forms a topological influence field over the network. By defining and computing the influence of the nodes with the distance $r=1$ and $r>1$ with target nodes. After a full consideration of the changes in data transmission and network topology caused by the variation of node amount, our approach can reflect and analyze the variation of node influence in different data reception periods. According to the influence results and contrast analysis in simulation experiments, compared with other methods, it can be easily applied to node classification, and the approach is able to make real-time dynamic evaluation on the node importance. The model presented in this paper is of flexibility which could be applied to some other research fields such as energy management and network survivability in the wireless sensor network in the future works.

\section{Acknowledgements}

The research presented in this paper is supported partly by the National Natural Science Foundation (Grant No:: 61172124, 61571360), the Shaanxi Science \& Technology Co-ordination \& Innovation Project (Grant No.: 2016KTZDGY05-09), and the Project of Xi'an Technology Bureau (No.: CXY1509-5).

\section{Authors' information}

Qindong Sun (corresponding author) received his Ph.D. degree at the School of Electronic and Information Engineering from the Xi'an Jiaotong University, China. He is currently the professor at the School of Computer Science and Engineering of the Xi'an University of Technology. His research interests include network information security, online social networks, and the Internet of things.

Yimin Qiao received his Master degree from the Xi'an University of Technology, China. He is currently a Ph.D. student at Xi'an University of Technology. His research interests include the Internet of things and data mining.

Jiamin Wang is currently the professor at the School of Mechanical and Precision Instrument Engineering of the Xi'an University of Technology. His research interests include product design and data mining.

Si Shen is currently a graduate student at the School of Computer Science and Engineering of the Xi'an University of Technology. Her research interests include the Internet of things and online social networks.

\section{Competing interests}

The authors declare that they have no competing interests.

\section{Author details}

'School of Computer Science and Engineering, Xi'an University of Technology, Xi'an 710048, China. ${ }^{2}$ Shaanxi Key Laboratory of Network Computing and Security, Xi'an University of Technology, Xi'an 710048, China. ${ }^{3}$ School of Mechanical and Precision Instrument Engineering, Xi'an University of Technology, Xi'an 710048, China.

Received: 31 May 2016 Accepted: 9 August 2016

Published online: 26 August 2016

\section{References}

1. FL Lewis, Wireless sensor networks. Smart Environments: Technologies, Protocols, and Applications 18(1), 11-46 (2005)

2. J Yick, B Mukherjee, D Ghosal, Wireless sensor network survey. Comput. Netw. 52(12), 2292-2330 (2015)

3. M Kuorilehto, M Hännikäinen, TD Hämäläinen, A survey of application distribution in wireless sensor networks [J]. EURASIP J. Wirel. Commun. Netw. 2005(5), 1-15 (2005)

4. Y Zhang, W Li, Modeling and energy consumption evaluation of a stochastic wireless sensor network [J]. EURASIP J. Wirel. Commun. Netw. 2012, 282 (2012)

5. L Mainetti, L Patrono, A Vilei, Evolution of wireless sensor networks towards the internet of things: a survey. Software Telecommunications and Computer Networks (SoftCOM) 2011, 1-6 (2011)

6. IF Akyildiz, W Su, Y Sankarasubramaniam, Wireless sensor networks: a survey Comput. Netw. 38(4), 393-422 (2002)

7. W Jia-sheng, W Xiao-ping, Y Bo, Improved method of node importance evaluation based on node contraction in complex networks. Procedia Eng. 15(1), 1600-1604 (2011)

8. D Chen, L Lü, MS Shang, Identifying influential nodes in complex networks[J]. Fuel Energy Abstr 391(4), 1777-1787 (2012) 
9. X Zhang, J Zhu, Q Wang, Identifying influential nodes in complex networks with community structure [J]. Knowl.-Based Syst. 42(2), 74-84 (2013)

10. Y Du, C Gao, Y Hu, A new method of identifying influential nodes in complex networks based on TOPSIS. Physica A. 399(4), 57-69 (2014)

11. T Agryzkov, JL Oliver, $L$ Tortosa, An algorithm for ranking the nodes of an urban network based on the concept of PageRank vector. Appl. Math. Comput. 219(4), 2186-2193 (2012)

12. $D$ Wei, $X$ Deng, $X$ Zhang, Identifying influential nodes in weighted networks based on evidence theory. Physica A. 392(10), 2564-2575 (2013)

13. H Nan, WY Gan, LD Yi, Evaluate nodes importance in the network using data field theory. Proceedings of the 2007 International Conference on Convergence Information Technology. IEEE Computer Society 2007, 1225$1234(2007)$

14. B Liu, YJ Sun, HR Liu, Key node decision algorithm based on local information and energy factor in wireless sensor network. J. of Commun. 36(10), 9-16 (2015)

15. E Goldfain, Complexity in quantum field theory and physics beyond the standard model. Chaos, Solitons Fractals 28(4), 913-922 (2006)

16. Q Sun, N Wang, Y Zhou, Modeling for user interaction by influence transfer effect in online social networks. IEEE 39th Conference on Local Computer Networks (LCN), IEEE Computer Society, 486-489(2014)

17. WR Heinzelman, A Chandrakasan, H Balakrishnan, Energy-efficient communication protocol for wireless sensor networks. Ad Hoc Sens. Wirel. Ne. 18, 8020 (2016)

18. O Younis, M Krunz, S Ramasubramanian, Node clustering in wireless sensor networks: recent developments and deployment challenges. IEEE Netw. 20(3), 20-25 (2010)

19. GV Merrett, NM White, NR Harris, Energy-aware simulation for wireless sensor networks. Sensor, Mesh and ad hoc communications and networks, 2009. SECON'09. 6th Annual IEEE Communications Society Conference on 2009, 1-8 (2009)

\section{Submit your manuscript to a SpringerOpen ${ }^{\mathcal{O}}$ journal and benefit from:}

- Convenient online submission

- Rigorous peer review

- Immediate publication on acceptance

- Open access: articles freely available online

- High visibility within the field

- Retaining the copyright to your article

Submit your next manuscript at $\gg$ springeropen.com 